\title{
FINITE CYCLIC SUBGROUPS DETERMINE THE SPECTRUM OF THE EQUIVARIANT $K$-THEORY
}

\author{
AGNIESZKA BOJANOWSKA
}

(Communicated by Frederick R. Cohen)

\begin{abstract}
Equivariant maps inducing an equivalence of the categories of components of the fixed point sets of topologically cyclic subgroups are considered. It is shown that they are the same as those inducing an equivalence of the categories of components of the fixed point sets of finite cyclic subgroups. It follows that equivariant maps inducing a bijection of maximal ideals of the appropriate equivariant $K$-theory rings coincide with those which give bijection on the sets of all prime ideals. As a corollary we obtain that a group homomorphism inducing bijection of maximal ideals of the representation rings is an isomorphism.
\end{abstract}

In this paper we study equivariant maps of spaces with group action from the point of view of the equivariant $K$-theory. Let $G$ and $G^{\prime}$ be compact Lie groups and $X, X^{\prime}$ be $G$ and $G^{\prime}$ spaces respectively. Throughout this paper we assume that all spaces are compact equivariant ENR's (cf. [tD, 5.2]). An equivariant map between $X$ and $X^{\prime}$, denoted $(\theta, f):(G, X) \rightarrow\left(G^{\prime}, X^{\prime}\right)$, consists of a homomorphism $\theta: G \rightarrow G^{\prime}$ and a continuous map $f: X \rightarrow X^{\prime}$ such that for every $x \in X, f(g x)=\theta(g) f(x)$. To a $G$ space $X$ we associate the category $C(G, X)$ which describes the components of the fixed point sets of topologically cyclic subgroups of $G$. By a topologically cyclic subgroup we mean a closure of a subgroup generated by one element. We recall the definition of $C(G, X)$ (cf. [Q, B]).

1. Definition. The objects of $C(G, X)$ are pairs $(S, c)$, where $S$ is a topologically cyclic subgroup of $G$ and $c \subset X^{S} \neq \varnothing$ is a connected component;

$$
\operatorname{Mor}_{C(G, X)}\left(\left(S_{1}, c_{1}\right),\left(S_{2}, c_{2}\right)\right)=\left\{g \in G: g S_{1} g^{-1} \subseteq S_{2}, g c_{1} \supseteq c_{2}\right\} / \approx,
$$

where $g \approx g^{\prime}$ iff for every $s \in S_{1}, g s g^{-1}=g^{\prime} s g^{\prime-1}$. We will denote by $[g]$ a morphism defined by $g \in G$.

The assignment to a $G$-space $X$ a category $C(G, X)$ is natural since a map $(\theta, f):(G, X) \rightarrow\left(G^{\prime}, X^{\prime}\right)$ defines a functor $(\theta, f)_{\#}: C(G, X) \rightarrow C\left(G^{\prime}, X^{\prime}\right)$ by means of the formulas: $(\theta, f)_{\#}(S, c)=(\theta S, f c),(\theta, f)_{\#}[g]=[\theta g]$, where

Received by the editors November 22, 1989.

1980 Mathematics Subject Classification (1985 Revision). Primary 55N91, 55N15, 22 E99. 
by $f c$ we denote the connected component of $X^{\theta S}$ containing $f(c)$. The purpose of the present paper is to prove that to check if $(\theta, f)_{\#}$ is an equivalence it suffices to look at the fixed-point sets of finite cyclic subgroups of $G$ and $G^{\prime}$.

Let $C F(G, X)$ denote the full subcategory of $C(G, X)$ consisting of all objects $(S, c) \in C(G, X)$ such that $S$ is a finite cyclic subgroup of $G$. If $(\theta, f)_{\#}: C(G, X) \rightarrow C\left(G^{\prime}, X^{\prime}\right)$ is an equivalence, then clearly $(\theta, f)_{\#}$ : $C F(G, X) \rightarrow C F\left(G^{\prime}, X^{\prime}\right)$ also is an equivalence. We prove that the converse is also true (Theorem 8 ).

The problem is related to studying equivariant maps from the point of view of the equivariant $K$-theory. A map $(\theta, f):(G, X) \rightarrow\left(G^{\prime}, X^{\prime}\right)$ induces a ring homomorphism $(\theta, f)^{*}: K_{G^{\prime}}\left(X^{\prime}\right) \rightarrow K_{G}(X)$ of the corresponding equivariant $K$-theory rings, which in turn defines a map of their prime ideal spectra $(\theta, f)_{*}: \operatorname{Spec} K_{G}(X) \rightarrow \operatorname{Spec} K_{G^{\prime}}\left(X^{\prime}\right)$. The maps $(\theta, f):(G, X) \rightarrow\left(G^{\prime}, X^{\prime}\right)$ inducing a homeomorphism $(\theta, f)_{*}: \operatorname{Spec} K_{G}(X) \rightarrow \operatorname{Spec} K_{G^{\prime}}\left(X^{\prime}\right)$ are characterized as those inducing an equivalence of categories $(\theta, f)_{\#}: C(G, X) \rightarrow$ $C\left(G^{\prime}, X^{\prime}\right)$, [B, Theorem 5.2]. Hence in particular we may conclude that any map inducing an equivalence $(\theta, f)_{\#}: C F(G, X) \rightarrow C F\left(G^{\prime}, X^{\prime}\right)$ induces a homeomorphism $(\theta, f)_{*}: \operatorname{Spec} K_{G}(X) \rightarrow \operatorname{Spec} K_{G^{\prime}}\left(X^{\prime}\right)$. The corollaries to Theorem 8 are formulated as Theorem 9 and Corollary 10.

The proof of the main result depends on an approximation of a topologically cyclic group by its finite cyclic subgroups.

2. Definition. For a given object $(S, c) \in C(G, X)$ we will say that a sequence $\left(S_{n}, c_{n}\right) \in C F(G, X)$ approximares $(S, c)$ iff $S_{n}$ is an ascending sequence of finite cyclic subgroups of $S$ whose union is dense in $S$ and $c_{n}=c$ for all $n$.

3. Proposition. Let $X$ be a compact G-ENR. For every $(S, c) \in C(G, X)$ there exists a sequence $\left(S_{n}, c_{n}\right) \in C F(G, X)$ approximating $(S, c)$.

Proof. If $S$ is a topologically cyclic group then $S=T^{m} \times C_{k}$, where $T^{m}$ is a torus group and $C_{k}$ is a cyclic group of order $k$. Let $p_{1}, \ldots, p_{m}$ be distinct primes relatively prime to $k$. The group $S_{n}=C_{p_{1}^{n}} \times \cdots \times C_{p_{m}^{n}} \times C_{k}$ is then cyclic. Clearly $X^{S_{1}} \supseteq X^{S_{2}} \supseteq \cdots \supseteq X^{S}$. Since $X$ is a compact $G$-ENR this sequence stabilizes, hence there exists $S_{l}$ such that $X^{S_{l}}=X^{S}$. The sequence $\left(S_{n+l}, c\right)$ is an approximation to $(S, c)$.

From now on we will assume that the condition $(*)$ holds

$$
\begin{aligned}
& \text { A map }(\theta, f):(G, X) \rightarrow\left(G^{\prime}, X^{\prime}\right) \text { induces an equivalence }(\theta, f)_{\#} \text { : } \\
& C F(G, X) \rightarrow C F\left(G^{\prime}, X^{\prime}\right) \text {. }
\end{aligned}
$$

4. Lemma. Let $\left(S^{\prime}, c^{\prime}\right) \in C\left(G^{\prime}, X^{\prime}\right)$. Then there exists an element $g^{\prime} \in G^{\prime}$ such that $\left(g^{\prime} S^{\prime} g^{\prime-1}, g^{\prime} c^{\prime}\right) \in \operatorname{im}(\theta, f)_{\#}$.

Proof. Let $\left(S_{n}^{\prime}, c^{\prime}\right) \in C F\left(G^{\prime}, X^{\prime}\right)$ be a sequence approximating $\left(S^{\prime}, c^{\prime}\right)$. We will inductively define a sequence of objects $\left(S_{n}, c_{n}\right) \in C F(G, X)$, for which 
$S_{n-1} \subseteq S_{n}, c_{n} \subseteq c_{n-1}$ and such that all subsets of $G^{\prime}$ defined by the formula:

$$
A_{n}=\left\{g^{\prime} \in A_{n-1}:\left(g^{\prime} S_{n}^{\prime} g^{\prime-1}, g^{\prime} c^{\prime}\right)=(\theta, f)_{\#}\left(S_{n}, c_{n}\right)\right\}
$$

will be nonempty.

By $(*)$ there exists $\left(S_{1}, c_{1}\right) \in C F(G, X)$ such that $(\theta, f)_{\#}\left(S_{1}, c_{1}\right)$ is isomorphic to $\left(S_{1}^{\prime}, c^{\prime}\right)$ in $C F\left(G^{\prime}, X^{\prime}\right)$. Let

$$
A_{1}=\left\{g^{\prime} \in G^{\prime}:\left(g^{\prime} S_{1}^{\prime} g^{\prime-1}, g^{\prime} c^{\prime}\right)=(\theta, f)_{\#}\left(S_{1}, c_{1}\right)\right\} \neq \varnothing .
$$

Now assume that the sequence has been defined up to $n-1$. Let $\left(\widehat{S}_{n}, \hat{c}_{n}\right)$ be any object such that $(\theta, f)_{\#}\left(\widehat{S}_{n} \hat{c}_{n}\right)=\left(h S_{n}^{\prime} h^{-1}, h c^{\prime}\right)$ for some $h \in G^{\prime}$. By the assumption $(*)$ :

$$
\begin{aligned}
& \operatorname{Mor}_{C F(G, X)}\left(\left(S_{n-1}, c_{n-1}\right),\left(\widehat{S}_{n}, \hat{c}_{n}\right)\right) \\
& \quad=\operatorname{Mor}_{C F\left(G^{\prime}, X^{\prime}\right)}\left((\theta, f)_{\#}\left(S_{n-1}, c_{n-1}\right),(\theta, f)_{\#}\left(\widehat{S}_{n}, \hat{c}_{n}\right)\right) \\
& \quad=\operatorname{Mor}_{C F\left(G^{\prime}, X^{\prime}\right)}\left(\left(g^{\prime} S_{n-1}^{\prime} g^{\prime-1}, g^{\prime} c^{\prime}\right),\left(h S_{n}^{\prime} h^{-1}, h c^{\prime}\right)\right)
\end{aligned}
$$

for some chosen $g^{\prime} \in A_{n-1}$.

Let $g \in G$ be such that $[g] \in \operatorname{Mor}_{C F(G, X)}\left(\left(S_{n-1}, c_{n-1}\right),\left(\widehat{S}_{n}, \hat{c}_{n}\right)\right)$ and $[\theta(g)]=\left[h g^{\prime-1}\right]$. Set $\left(S_{n}, c_{n}\right)=\left(g^{-1} \widehat{S}_{n} g, g^{-1} \hat{c}_{n}\right)$. Then $(\theta, f)_{\#}\left(S_{n}, c_{n}\right)=$ $\left(\theta\left(g^{-1}\right) h S_{n}^{\prime} h^{-1} \theta(g), \theta\left(g^{-1}\right) h c^{\prime}\right)$ is isomorphic to $\left(S_{n}^{\prime}, c^{\prime}\right)$ and $S_{n-1} \subseteq S_{n}$, $c_{n} \subseteq c_{n-1}$. The set $A_{n}$ defined by $\left(S_{n}, c_{n}\right)$ is nonempty because $\left[\theta\left(g^{-1}\right) h\right]=$ $\left[g^{\prime}\right]$ on $\left(S_{n-1}^{\prime}, c^{\prime}\right)$ and hence $\theta\left(g^{-1}\right) h \in A_{n}$.

It is obvious that $A_{n}$ are closed, form a descending sequence, and therefore $\bigcap_{n} A_{n} \neq \varnothing$. Let $S=\bigcup_{n} S_{n}$. Then $S$ is topologically cyclic and $c=\bigcap_{n} c_{n}$ is nonempty. It is clear that for any element $g^{\prime}$ from $\bigcap_{n} A_{n},(\theta, f)_{\#}(S, c)=$ $\left(g^{\prime} S^{\prime} g^{\prime-1}, g^{\prime} c^{\prime}\right)$.

Observe that $(*)$ implies the following property of $\theta$ :

5. Lemma. If $(\theta, f)_{\#}: C F(G, X) \rightarrow C F\left(G^{\prime}, X^{\prime}\right)$ is an equivalence, then for every topologically cyclic subgroup $S \subseteq G$ such that $X^{S} \neq \varnothing$, the homomorphism $\theta$ restricted to $S$ is a monomorphism.

Proof. Suppose that $\operatorname{ker}\left(\left.\theta\right|_{S}\right) \neq\{1\}$. Let $S_{1} \subseteq S$ be a finite nontrivial cyclic subgroup contained in $\operatorname{ker}\left(\left.\theta\right|_{S}\right)$ and let $c_{1}$ be a connected component of $X^{S_{1}}$. Let $c$ be the connected component of $X$ containing $c_{1}$. Then the induced map

$$
\operatorname{Mor}_{C F(G, X)}\left(\left(S_{1}, c_{1}\right),(\{1\}, c)\right) \rightarrow \operatorname{Mor}_{C F\left(G^{\prime}, X^{\prime}\right)}\left(\left(\theta S_{1}, f c_{1}\right),(\{1\}, f c)\right)
$$

is a bijection. But this is impossible since the first set is empty and the second consists of one element. Hence $\operatorname{ker}\left(\left.\theta\right|_{S}\right)=\{1\}$.

To prove that $(\theta, f)_{\#}: C(G, X) \rightarrow C\left(G^{\prime}, X^{\prime}\right)$ is an equivalence we still must show that for arbitrary $\left(S_{1}, c_{1}\right),\left(S_{2}, c_{2}\right) \in C(G, X)$, the induced map:

$$
\operatorname{Mor}_{C(G, X)}\left(\left(S_{1}, c_{1}\right),\left(S_{2}, c_{2}\right)\right) \rightarrow \operatorname{Mor}_{C\left(G^{\prime}, X^{\prime}\right)}\left(\left(\theta S_{1}, f c_{1}\right),\left(\theta S_{2}, f c_{2}\right)\right)
$$


is a bijection. We first show that it is surjective.

6. Lemma. For every morphism [ $\left.g^{\prime}\right]:\left(\theta S_{1}, f c_{1}\right) \rightarrow\left(\theta S_{2}, f c_{2}\right)$ there exists a morphism $[g]:\left(S_{1}, c_{1}\right) \rightarrow\left(S_{2}, c_{2}\right)$ such that morphisms $[\theta g]$ and $\left[g^{\prime}\right]$ are equal.

Proof. Let $\left(\widetilde{S}_{n}, c_{1}\right), n \in N$ be a sequence approximating $\left(S_{1}, c_{1}\right)$. We have then $g^{\prime} \theta\left(\widetilde{S}_{n}\right) g^{\prime-1} \subseteq \theta\left(S_{2}\right)$. We define a decreasing sequence $A_{n} \supseteq A_{n+1}$ of closed subsets of $G$ :

$$
A_{n}=\left\{g \in G:[\theta g]=\left[g^{\prime}\right]:\left(\theta \widetilde{S}_{n}, f c_{1}\right) \rightarrow\left(\theta S_{2}, f c_{2}\right)\right\} .
$$

We must prove that each $A_{n}$ is nonempty. Let $\widehat{S}_{n}$ be $S_{2} \cap \theta^{-1}\left(g^{\prime} \theta\left(\widetilde{S}_{n}\right) g^{\prime-1}\right)$. Then by Lemma $5, \widehat{S}_{n}$ is finite and $\theta\left(\widehat{S}_{n}\right)=g^{\prime} \theta\left(\widetilde{S}_{n}\right) g^{\prime-1} \subseteq \theta\left(S_{2}\right)$. If $\hat{c}_{n}$ is a connected component of $X^{\widehat{S}_{n}}$ containing $c_{2}$ then $\left[g^{\prime}\right]$ defines a morphism $\left(\theta \widetilde{S}_{n}, f c_{1}\right) \rightarrow\left(\theta \widehat{S}_{n}, f \hat{c}_{n}\right)$. By $(*)$ there exists $g \in G$ such that $[\theta g]=\left[g^{\prime}\right]$ as morphisms $\left(\theta \tilde{S}_{n}, f c_{1}\right) \rightarrow\left(\theta \widehat{S}_{n}, f \hat{c}_{n}\right)$, so $g \in A_{n}$. Hence $\bigcap_{n} A_{n} \neq \varnothing$ and for any element $g \in \bigcap_{n} A_{n}$ we have $[\theta g]=\left[g^{\prime}\right]$ as morphisms $\left(\theta S_{1}, f c_{1}\right) \rightarrow$ $\left(\theta S_{2}, f c_{2}\right)$.

We next prove:

7. Lemma. For every $\left(S_{1}, c_{1}\right),\left(S_{2}, c_{2}\right) \in C(G, X)$ the map $(\theta, f)_{\# \text {, }}$,

$$
\operatorname{Mor}_{C(G, X)}\left(\left(S_{1}, c_{1}\right),\left(S_{2}, c_{2}\right)\right) \rightarrow \operatorname{Mor}_{C(G, X)}\left(\left(\theta S_{1}, f c_{1}\right),\left(\theta S_{2}, f c_{2}\right)\right.
$$

is injective.

Proof. This is an immediate consequence of Lemma 5.

Lemmas 4, 6, and 7 complete the proof of the following theorem.

8. Theorem. An equivariant map $(\theta, f):(G, X) \rightarrow\left(G^{\prime}, X^{\prime}\right)$ induces an equivalence $C F(G, X) \rightarrow C F\left(G^{\prime}, X^{\prime}\right)$ if and only if it induces an equivalence $C(G, X) \rightarrow C\left(G^{\prime}, X^{\prime}\right)$.

There is a correspondence between maximal ideals and objects of $C F(G, X)$ described in [B, Proposition 4.7]. It leads to a natural homeomorphism $\alpha$ : indlim ${ }_{C F(G, X)} \operatorname{MSpec} R(S) \rightarrow \operatorname{MSpec} K_{G}(X)$, [B, Theorem 3.7, Lemma 4.2]. This enables us to repeat almost word by word part of [B, Proof of Theorem 5.2] and obtain the following:

9. Theorem. Let $(\theta, f):(G, X) \rightarrow\left(G^{\prime}, X^{\prime}\right)$ be an equivariant map. Then the following conditions are equivalent :

(a) $(\theta, f)_{*}: \operatorname{Spec} K_{G}(X) \rightarrow \operatorname{Spec} K_{G^{\prime}}\left(X^{\prime}\right)$ is a bijection;

(b) $(\theta, f)_{*}: \operatorname{MSpec} K_{G}(X) \rightarrow \operatorname{MSpec} K_{G^{\prime}}\left(X^{\prime}\right)$ is a bijection;

(c) $(\theta, f)_{\#}: C(G, X) \rightarrow C\left(G^{\prime}, X^{\prime}\right)$ is an equivalence;

(d) $(\theta, f)_{\#}: C F(G, X) \rightarrow C F\left(G^{\prime}, X^{\prime}\right)$ is an equivalence. 
A result similar in spirit to the equivalence of (a) and (d) was obtained by J. McClure (cf. [Mc]).

In the case $X=X^{\prime}=p t$ one can prove that a homomorphism $\theta: G \rightarrow G^{\prime}$ inducing an equivalence of categories $C(G) \rightarrow C\left(G^{\prime}\right)$ must be an isomorphism (cf. [B, M]). Hence we obtain:

10. Corollary. If $\theta: G \rightarrow G^{\prime}$ is a homomorphism of compact Lie groups inducing a bijection of maximal ideals of their complex representation rings then $\theta$ is an isomorphism.

\section{REFERENCES}

[B] A. Bojanowska, The spectrum of equivariant $K$-theory, Math. Z. 183 (1983), 1-19.

[tD] T. Tom Dieck, Transformation groups and representation theory, Lecture Notes in Math., vol. 766, Springer-Verlag, Berlin, Heidelberg, and New York, 1979.

[M] N. Minami, Group homomorphisms inducing an isomorphism of a functor, Math. Proc. Cambridge Philos. Soc. 104 (1990), 81-93.

[Mc] J. McClure, Restriction maps in equivariant K-theory, Topology 25 (1986), 399-411.

[Q] D. Quillen, The spectrum of equivariant cohomology ring I, II, Ann. of Math. 94 (1971), 549-602.

Uniwersytet Warszawski, Instytut Matematyki, Ul. Banacha 2, Pl-00-913 Warszawa 59, Poland 